\title{
Comparison of Triosil 370 with Urografin 76 and Hypaque 65 for coronary arteriography
}

\author{
D. Verel, C. Ward, and M. Aman \\ From the Cardiothoracic Unit, Northern General Hospital, Sheffield
}

Triosil 370 has been compared with Urografin 76, and Hypaque 65 by injecting identical volumes into the coronary arteries of human subjects in the course of coronary arteriography. The injection times used were the same and neither the patient nor the coronary artery catheter were moved between injections. The order in which the solutions compared were injected was alternated. The deviation in the ST segment and of the T wave which resulted from the injection was measured. The Triosil caused significantly less change than Urografin and Hypaque. A numerical expression of the $T$ wave deflection derived from the data showed Triosil: Hypaque: Urografin $=I: 2.6: 2.9$.

The development of successful operations for the alleviation of angina pectoris has resulted in a great increase in coronary arteriography. This investigation carries a risk of ventricular arrhythmias which is related to a number of factors of which one is the nature of the contrast medium injected into the coronary artery. All injected contrast media consist of organic iodine compounds, and the balance of the cations in solution has been shown to be of particular importance in determining the suitability for coronary artery injection. Much preliminary work has been done on the coronary circulation of experimental animals but trials on man are necessary since it has been shown that the responses of the experimental animal and of man differ significantly (Ovitt et al., 1972). The response to perfusion of the coronary arteries may be measured by examination of the effect on the electrocardiogram, by measurement of the constituents of the coronary sinus blood, and by determining the effect on such factors as cardiac output, peripheral vascular resistance, and many other parameters. Most of these are easily applied in the laboratory to experimental animals, but in man during the performance of diagnostic coronary arteriography only the examination of the effect on the electrocardiogram has been widely used (Eie et al., 1972; Coskey and Magidson, 1967).

In this study we have compared the effects of Triosil 370 with those of Urografin 76. These solutions have identical iodine concentrations of $370 \mathrm{mg} / \mathrm{ml}$.

Received 3 March 1975.

\section{Methods}

All patients were investigated for suspected coronary artery disease using Judkins catheters introduced by Seldinger technique into the femoral artery.

Coronary catheter pressure was monitored continuously by strain gauge. The gauge was connected by a three-way tap which was in turn connected to a $60 \mathrm{~cm}$ transparent plastic manometer connector (Portland Plastics), ending in a second three-way tap to which the Judkins catheter was connected. All injections of contrast medium were made into this second three-way tap. Patients with severe myocardial dysfunction were excluded by a preliminary left ventricular angiocardiogram. Comparisons were only made in patients in whom the coronary catheter could be introduced into the coronary artery without causing a fall in pressure to below $85 \mathrm{mmHg}(\mathrm{Ir} .3 \mathrm{kPa})$ systolic. When a comparison was made, the three-way tap between the Judkins catheter and the manometer connector tube was turned so that all three ways were open. A measured volume $(4$ to $8 \mathrm{ml})$ of contrast medium was injected. The deflection on the pressure record was used to measure the injection time (Fig. 1). The electrocardiogram was recorded continuously for the duration of the injection and for the ensuing 15 to 30 seconds.

The electrocardiogram was observed until it had returned to its preinjection form. After waiting a further three minutes a second injection of a different contrast medium was then made using the same volume and injection time and without altering the position of either the patient or the catheter. Comparisons were not attempted if the electrocardiogram failed to return to its previous form. In repeated comparisons the order in which the contrast media were injected was alternated. Since it was usual to take 4 projections of the left coronary artery often on single and double image, and 2 of the right, similarly on single and double image, it was 

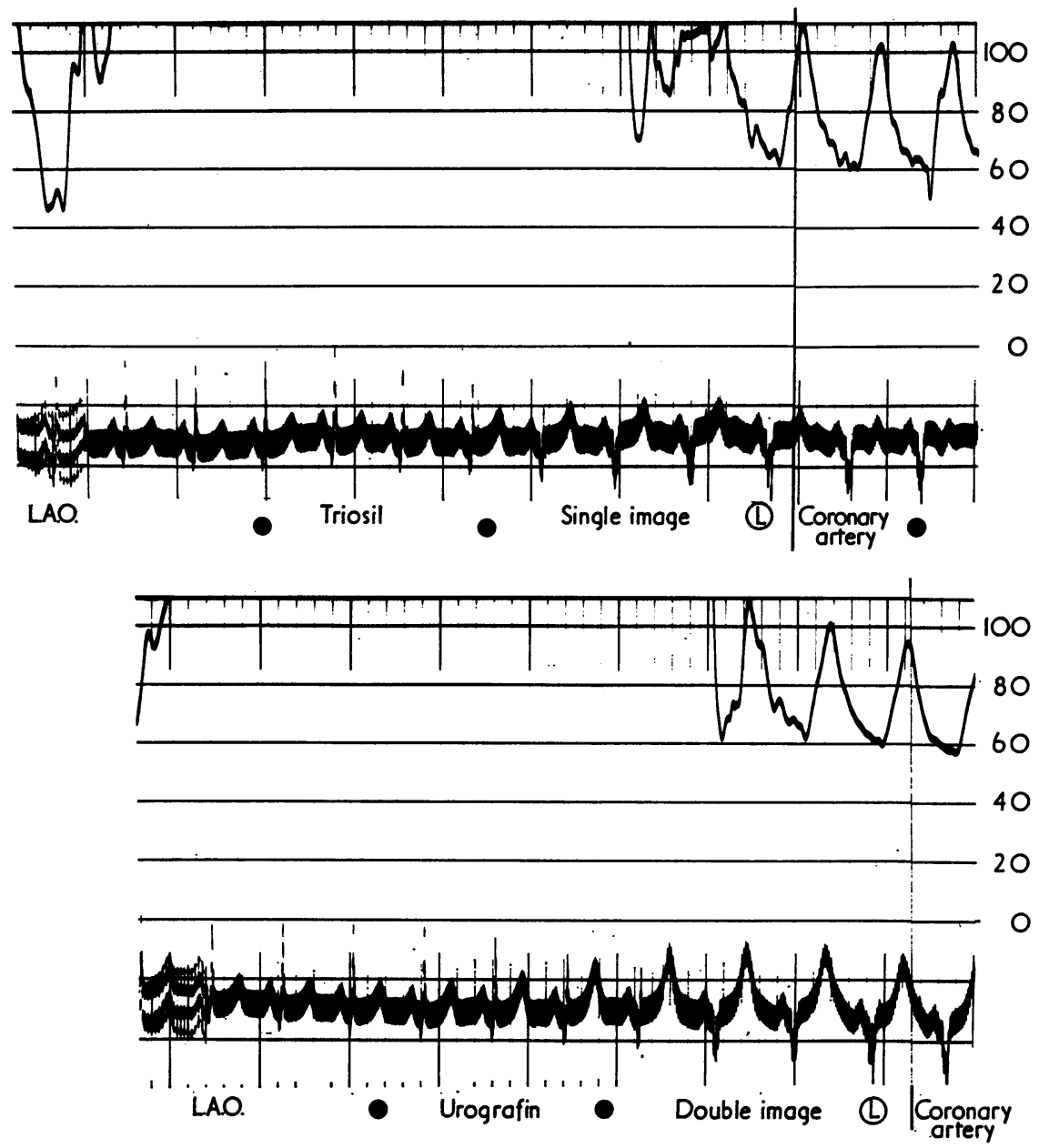

FIG. I Comparison of Triosil 370 (top) and Urografin 76 (bottom). The injection is signalled by the upward movement of the coronary artery pressure trace off the recording paper. Note the greater $T$ wave change in the Urografin record.

possible to get 6 comparisons from a single patient. The usual number, however, was much less since the comparisons were only made when single and double image views of the coronary artery were made, and in many patients no comparison was possible because the catheter had to be withdrawn at the end of the injection as it was occluding the artery. Such occlusion is itself a cause of electrocardiographic abnormality and would invalidate any comparison of the kind described.

At the end of the procedure the cardiac output was measured by dye dilution. All patients returned to a coronary care unit for some hours after the catheterizations.

The effects of the injection of contrast media were assessed by measuring the deflection of the $T$ wave in the electrocardiogram (Fig. I). Others using this technique have in addition measured the change in electrical axis and the duration of the electrocardiographic abnormality (Eie et al., I972). In our cases the duration of the electrocardiographic abnormality correlated well with the ST deviation but was more difficult to measure accurately. The change in the axis of the electrocardiogram seemed to be more closely related to the distribution of the injected artery than to the nature of the substance injected.

\section{Results}

The results of 20 comparisons of Urografin 76 with Triosil 370 in 5 patients are shown in Fig. 2. In two instances the electrocardiogram was unchanged by the injection of both contrast media. In one comparison (No. 3) the deflection caused by Triosil 370 was I mm greater than that caused by Urografin 76 ; in the remaining 17 comparisons the deflection caused by the Urografin exceeded that caused by 


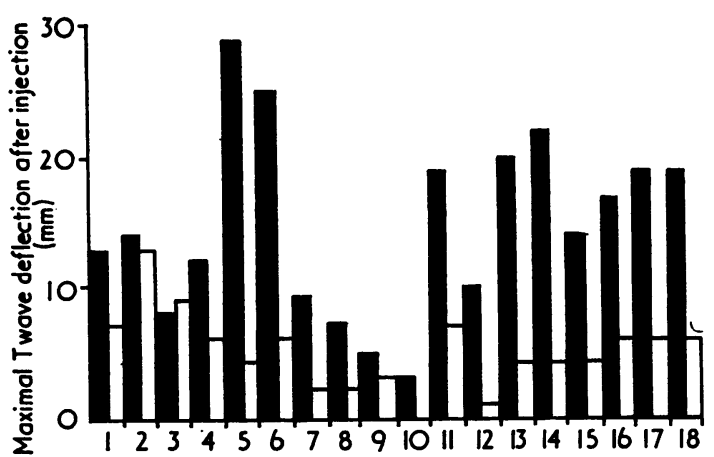

FIG. 2 Comparison of the maximal $T$ wave deflection following 20 pairs of injections of Triosil 370 and Urografin 76. Though the Urografin is shown on the left in each pair the order of injection was alternated so that each drug was injected first in half the cases.

Triosil. The total sum of the deflections caused by Urografin in 20 cases is $265 \mathrm{~mm}$, while that caused by Triosil is $90 \mathrm{~mm}$. This difference is highly significant, and indicates that Triosil 370 is less toxic in its effects on the human myocardium than Urografin 76.

A similar comparison between Triosil 370 and Hypaque 65 was then made, 20 comparisons being again obtained. In this series the sum of the deflections of the $T$ wave caused by Triosil 370 was I77 $\mathrm{mm}$ while that caused by Hypaque 65 was 463 $\mathrm{mm}$. These results are also significant, and indicate that Triosil 370 is a more physiological solution than Hypaque 65.

No arrhythmias occurred after the injection of any of these contrast media, but it should be noted that these injections were only made when the coronary artery pressure was high. Arrhythmias were observed occasionally with all these solutions when injections had to be made into arteries where the arterial pressure was low because of obstruction to the coronary ostium by the catheter.

\section{Discussion}

Comparison of the electrocardiographic abnormalities that result from the injection of solutions other than blood into the coronary arteries is widely accepted as a valid measurement of the toxicity of such solutions to the myocardium. The desirability of trials on man rather than on experimental animals has been shown by Ovitt et al. (1972). These authors found that normal saline produced similar adverse changes in the electrocardiogram in both dogs and man while Krebs-Hanseleit solution in both species caused little change. Dextrose solution ( $5 \%$ in water), however, caused striking abnormalities in the ST segment in dogs similar to those caused by saline, but in man little change occurred, the results resembling those produced by the Krebs-Hanseleit solution. No similar comparison between experimental animals and man appears to have been made.

The three solutions compared are all mixtures of salts of iodinated benzoic acid. The composition, iodine content, and physical characteristics are shown in the Table. It is evident that the differences encountered between these media are not caused by differences in viscosity or iodine content. In a series of comparisons of different contrast media it has been shown that the addition of small amounts of sodium and calcium salts to the iodine-containing molecule reduces the toxic effects which follow coronary artery injection (Jacobsson and Paulin, 1967; Bjork, 1967). A comparison of the three mixtures suggests that the addition of the calcium salt in Triosil 370 may be a critical factor.

The method of comparing injections made without moving the patient or the catheter was adopted because rotating the patient for a different $x$-ray projection causes major changes in the electrocardiographic axis, the direction of the $T$ wave, and the height of the ST segment (Verel and Grainger, 1973). This makes comparison of the ST deflection difficult if the patient is moved between injections. Similarly, if the coronary artery catheter is withdrawn and replaced between injections it is possible that the area of myocardium perfused by the injectate is not the same; it is not unusual to pass a Judkins catheter beyond an early branch of either coronary artery on one occasion and so fail to fill it with contrast medium, and to perfuse it on another occasion when the catheter is not passed so far in. Most previous authors making comparisons of the type described have waited up to 15 minutes before making a second injection. It is not possible to leave a coronary artery catheter in situ for this length of time, and we made injections at intervals of 3 to 5 minutes. Repeated injections of the same contrast medium given under these conditions showed similar ST deflections. In addition, the order in which the contrast media were given was alternated. The short period between the injections does not, therefore, invalidate the results.

It will be noted that 20 injections of Triosil caused a deflection of $90 \mathrm{~mm}$ in the first series, and of $177 \mathrm{~mm}$ in the second. These figures are not comparable as the electrocardiographic voltages were not standardized at catheterization. The electrocardiogram trace was adjusted to a convenient size which was retained throughout the catheterization. In addition, movements of the patient altered the $T$ 
TABLE Composition and characteristics of contrast media

\begin{tabular}{lllll}
\hline Solution & Iodine salts & & $\begin{array}{l}\text { Iodine } \\
\text { content } \\
(\mathrm{mg} / \mathrm{ml})\end{array}$ & $\begin{array}{l}\text { Viscosity } \\
\text { at } 37^{\circ} \mathrm{C}\end{array}$ \\
\hline Hypaque 65 & $\begin{array}{l}\text { Meglumine diatrizoate } \\
\text { Sodium diatrizoate }\end{array}$ & $\begin{array}{r}50 \% \\
25 \%\end{array}$ & 390 & 8.4 \\
Urcgrafin 76 & $\begin{array}{l}\text { Meglumine diatrizoate } \\
\text { Sodium diatrizoate } \\
\text { Meglumine metrizoate }\end{array}$ & $\begin{array}{r}65 \% \\
10 \%\end{array}$ & 370 & 8.5 \\
Triosil 370 & $\begin{array}{l}85 \% \\
\text { Sodium metrizoate } \\
\text { Calcium metrizoate }\end{array}$ & $\begin{array}{r}13 \% \\
2 \%\end{array}$ & 370 & 8.5 \\
\hline
\end{tabular}

wave voltage and direction. It follows that the comparisons of one contrast medium with another in a pair of injections is valid, but one injection in a pair of injections cannot be compared with a similar injection in another pair. It is possible, however, to compare the ratios obtained from the figures for total $T$ wave deflection. The two comparisons may be expressed thus: Triosil 370:Urografin $76=90: 265$ which equals I: 2.9. Triosil 370:Hypaque $65=$ 177:463 which equals I: 2.6, thus Triosil 370: Hypaque $65:$ Urografin $76=1: 2.6: 2.9$.

This method of expression, of course, does not imply that Hypaque 65 is 2.6 times as toxic as Triosil 370 , but only that it may be expected to produce 2.6 times as much shift in the $T$ wave after injection of a comparable volume in similar time. It should, however, provide a simple numerical basis for comparison of contrast media used for coronary artery injection.

\section{References}

Bjork, L. (1967). Further experiences with Isopaque B in angiocardiography and coronary angiography. Acta Radiologica, Suppl. 270, 197.

Coskey, R. L., and Magidson, O. (1967). Electrocardiographic response to selective coronary arteriography. British Heart fournal, 29, 512.

Eie, H., Grendahl, H., Nordvik, A., and Müller, C. (1972). Electrocardiographic changes during selective coronary arteriography. Acta Radiologica, 12, 554.

Jacobsson, B., and Paulin, S. (1967). Experiences with different contrast media in coronary angiography. Acta Radiologica, Suppl. 270, 194.

Ovitt, T., Rizk, G., Frech, R. S., Cramer, R., and Amplatz, K. (1972). Electrocardiographic changes in selective coronary arteriography; the importance of ions. Radiology, $104,705$.

Verel, D., and Grainger, R. G. (1973). Cardiac Catheterisation and Angiocardiography, and ed., p. 93. Churchill-Livingstone, Edinburgh and London.

Requests for reprints to Dr. D. Verel, Cardiothoracic Unit, Northern General Hospital, Sheffield S5 7AU. 